\title{
IMPROVING OF DATA PROCESSING EFFECTIVENESS FOR PAVEMENT STRUCTURAL EVALUATION USING SUBSURFACE RADAR PROBING
}

\author{
Alexander Krainyukov, Valery Kutev \\ Transport and Telecommunications Institute \\ Lomonosova str. 1, Riga, LV-1019, Latvia \\ Ph.: +37167100634.Fax: +37167100660 \\ E-mail: Krainukovs.A@tsi.lv,Kutevs.V@tsi.lv
}

Problems of the data processing improving for pavement structure evaluation with help of subsurface radar probing are discussed. Iterative procedure to solve the inverse problem in frequency domain is used on the base of the genetic algorithm. For improving of data processing effectiveness it is proposed to use a modified genetic algorithm with adaptation of search range of pavement parameters. The results of reconstruction of electro-physical characteristics for model of five-layered pavement structure are presented.

Keywords: pavement evaluation, subsurface radar probing, inverse problem, pavement parameter search range, genetic algorithm, adaptation of search range, pavement modelling

\section{Introduction}

Roads are subjected to heavy loadings and harsh environmental conditions, due to which they deteriorate with time. The rate of deterioration depends on the construction materials used, rate of loading, and environmental conditions. Therefore, road performance has to be monitored to evaluate the rate of deterioration and need for maintenance and rehabilitation. Pavement structural evaluation is concerned with the structural capacity of the pavement, layer thickness, and material properties. It is used to obtain information on the load-bearing capacity of roads to evaluate the need for maintenance and rehabilitation, asset pavement evaluation, and construction quality control.

The inner structure of typical pavement (Fig. 1) consists of some layers: surfacing, base, sub-base, improved sub-grade and sub-grade [1].

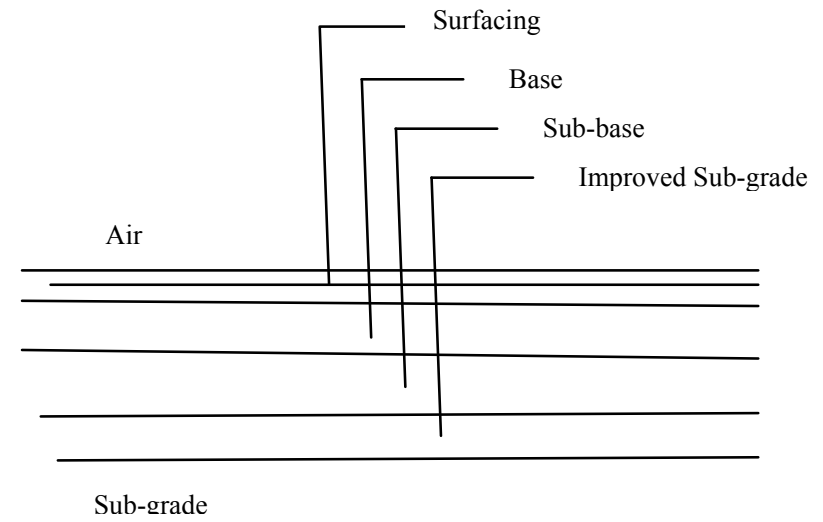

Figure 1. The inner structure of typical pavement

Surfacing is the riding surface of the road and varies from a light bitumen spray with stone chippings (bitumen surface treatment) to one or more layers of dense bitumen surfacing. Base is the main load-spreading layer of the road pavement. It must be constructed of high quality brick or stone aggregate. Sub-base is the secondary load-spreading layer of the pavement. It is usually either crushed stone, broken brick aggregate (frequently mixed with sand) or locally available gravel. Improved Sub-grade is imported material (e.g. fine or coarse sand) that is locally available. It is intended to act as a cushioning layer between weak sub-grades and the road pavement layers (if the sub-grade is of sufficient strength then an improved sub-grade may not be required.

Sub-grade is the soil immediately beneath the road structure. 
During assessment evaluation of pavement conditions information must be gathered about the surface of the road, the structural strength of the material used in the pavement and roadbed construction. Pavement condition evaluation and monitoring using traditional methods such as visual inspection are labour intensive, slow and expensive. More efficient and automated methodology for road pavement inspection by using ground penetrating radar (GPR) is proposed to identify the cause of existing problems and define optimal strategies for repair and rehabilitation.

GPR has been used extensively in the road pavement for quite some time and was performed in early 1980s. Most of the research and development works in road application have been performed with low frequency $(100-5000 \mathrm{MHz})$ to evaluate and survey the road pavement layers condition [3]. The majority of GPR applications resolves around the estimation of layer thickness using the travel-time technique, where the layer thickness is presented in form of so-called radar traces [2]. To provide the required effectiveness of structural evaluation it is necessary to perform reconstruction of electro-physical parameters of pavement layers. Such reconstruction can be achieved by solving the inverse problem of roadway subsurface radar probing [3].

\section{Inverse Problem of Pavement Subsurface Radar Probing}

To solving the inverse problem the comparison method may be used in which the results of the radar forward problem solution for an assumed subsurface structure with limited area of electro-physical parameters changing are comparing with data of radar probing. The comparison method may be performed both in the frequency domain and in the time domain. We used the comparison method for investigated the inverse problem of roadway coverage radar probing in the frequency domain $[4,5,7]$. The model-based approach for radar probing data inversion of roadway coverage in the frequency domain is presented on Figure 2.

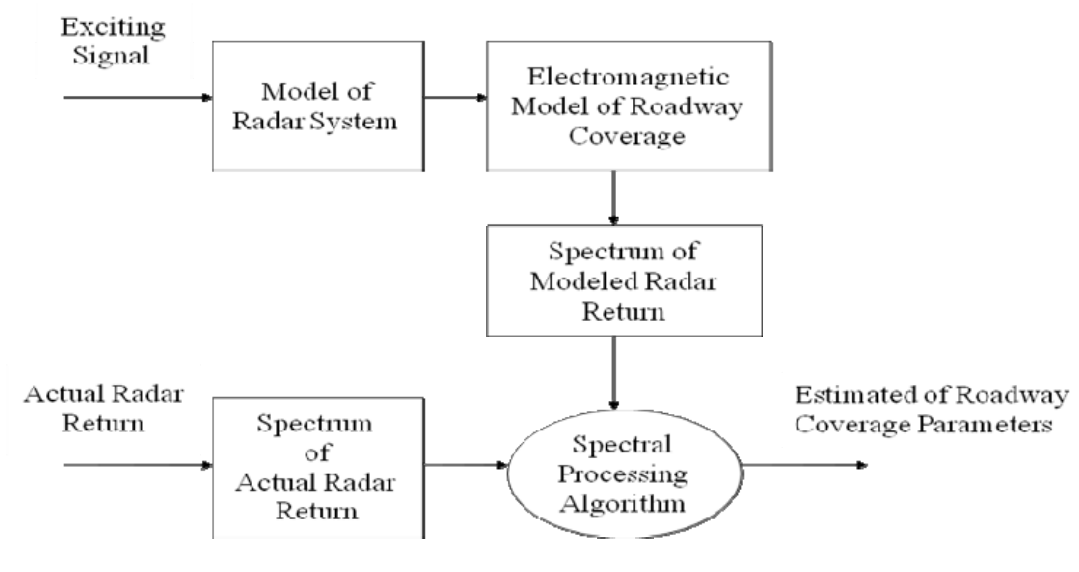

Figure.2. The model -based approach for radar monitoring data inversion

The initial stage of the iterative procedure is concerned with calculating of spectral density $\dot{S}_{e}\left(\omega_{i}, \vec{P}\right)$ for reflected signal $U_{r e f}(t)$. Further, in order to solve the inverse problem of subsurface radar probing, we introduce vector of parameters:

$\vec{P}=\left\{p_{1}, p_{2}, \ldots, p_{n}\right\} \in \vec{P}_{P O S}$,

where $\vec{P}_{P O S}$ - is a set of possible (allowed) values for parameters of probed medium. The set of allowed values for parameters is determined on the basis of pre-existing hypotheses about internal structure of probed medium. The component $p_{i}$ is electro-physical parameter of layers for $n$-layered model of probed media. Electro-physical parameters of each layer are as follows: layer thickness $h$, electrical conductivity $\sigma$ and relative dielectric permittivity $\varepsilon$ of the layer's materials.

Calculating of theoretical spectral density $\dot{S}_{T}\left(\omega_{i}, \vec{P}\right)$ equates to the solving of the direct problem of subsurface radar probing. To calculate $\dot{S}_{T}\left(\omega_{i}, \vec{P}\right)$ we chose the starting vector of parameters $\vec{P}_{M}$ belonging to the set of allowed values of parameters $\vec{P}_{P O S} \cdot \vec{P}_{\min }$ is vector of minimum values of the parameters of the probed medium, $\vec{P}_{\max }$ is the vector of maximum values of the parameters of the probed medium. 
Values of experimental and theoretical spectral densities are used to calculate aim function $\Phi$ :

$\Phi=\left\|\dot{S}_{e}\left(\omega_{i}, \vec{P}\right)-\dot{S}_{T}\left(\omega_{i}, \vec{P}_{M}\right)\right\|^{2}=\frac{1}{n_{\max }} \sum_{i=0}^{n_{\max }}\left|\dot{S}_{e}\left(\omega_{i}, \vec{P}\right)-\dot{S}_{T}\left(\omega_{i}, \vec{P}_{M}\right)\right|^{2}$

where $n_{\max }$ - index of the spectral component with frequency $f_{\max }$.

Block diagram of basic iterative procedure for solving inverse problem of pavement subsurface radar probing in frequency domain is shown on Figure 3.

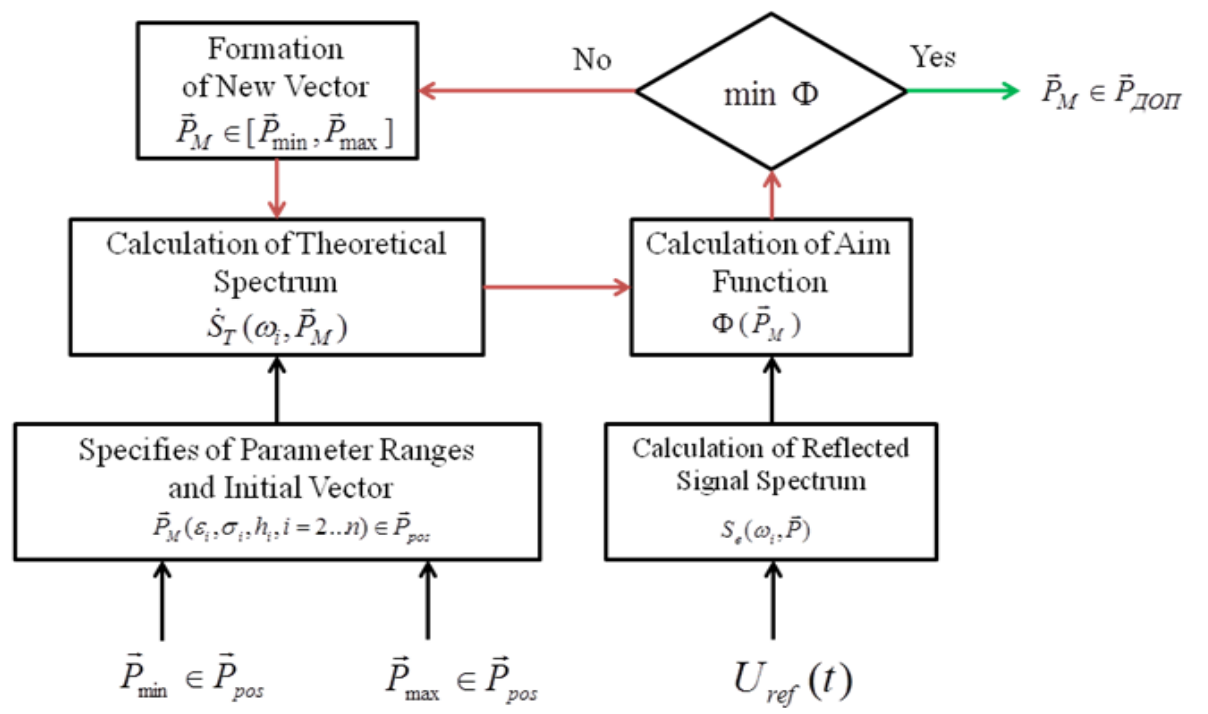

Figure 3. Basic iterative procedure for solving inverse problem of pavement subsurface radar probing

If the value of the aim function $\Phi$ is not more than the value of a threshold $\alpha$, then solving the inverse structure problem is finished.

The value of a threshold $\alpha$ is set as follows:

$\alpha=\frac{P_{a v}}{K}$,

where $P_{a v}$ - is the averaged mean power of those spectral components $\dot{S}_{e}\left(\omega_{i}, \vec{P}\right)$, which are used for calculating of aim function $\Phi$, and $K$-dimensionless coefficient, set by the user.

The solution (pseudo-solution) is the vector of parameters $\vec{P}_{M}$. If the value of the aim function $\Phi$ is greater than the value of $\alpha$, then taking into account the current values of the vector $\vec{P}_{M}$ we formulate a new vector of parameters $\vec{P}_{M}$, which is used to calculate new values of the aim function $\Phi$. This means, that finding a solution to the inverse problem in the form of the vector $\vec{P}_{M}$ is performed by iteration with the sequential improvement of accuracy of the parameters $\vec{P}_{M}$.

\section{Modification of Genetic Algorithm for Solution of Inverse Problem}

Inverse problem of pavement subsurface radar probing can't be solved exactly within bounded computation times. This generates much interest in search algorithms that find near-optimal solutions in reasonable running times.

The modified genetic algorithm with the adaptive narrowing parameter's search range is a search algorithm capable of locating good solutions efficiently. The algorithm could be regarded as belonging to the category of "intelligent" optimisation tools [6].

Block diagram of iterative procedure to solve radar inverse problem of pavement subsurface radar probing with using of modified genetic algorithm is shown in Figure 4. 


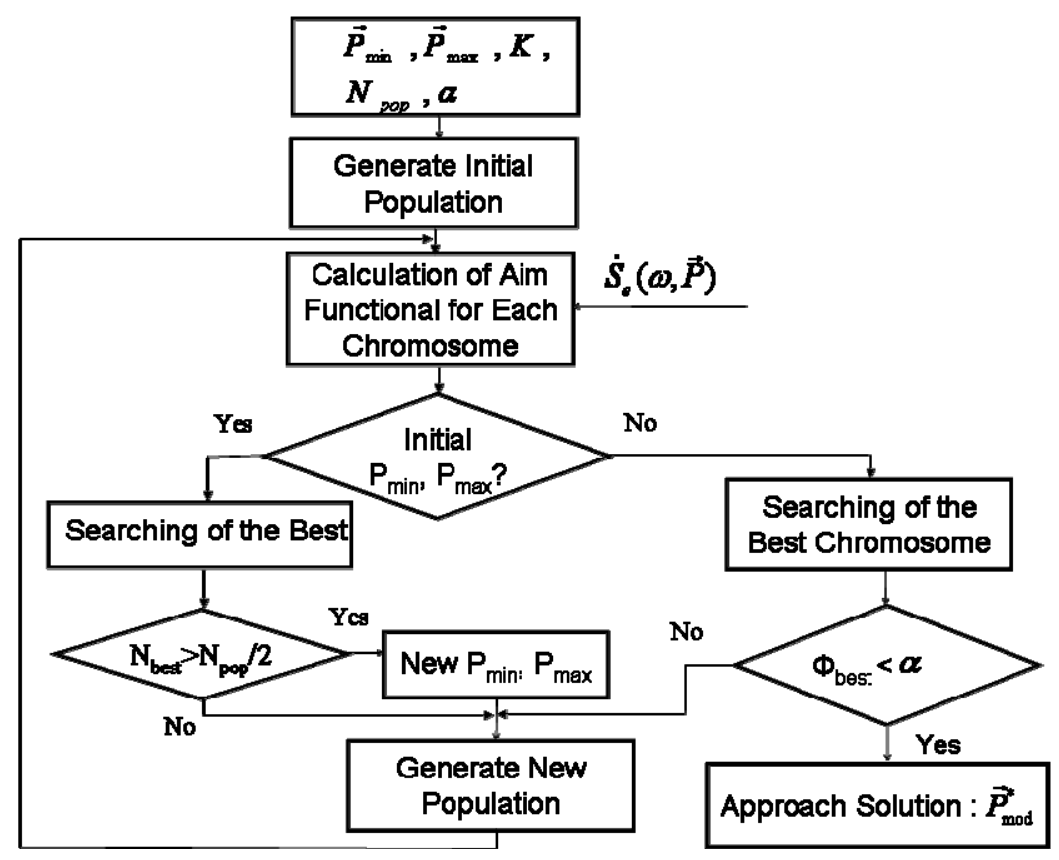

Figure 4. Block diagram of modified genetic algorithm

In the first stage the values of the search range $\vec{P}_{\min }$ and $\vec{P}_{\max }$ are determined a priori information about the probed roadway. Values $K$ и $\mathrm{N}_{\text {рор }}$ (population size) are set by the user in beginning of solving the inverse problem. Therefore we refined the search ranges in the first stage, when the $K$ value was low.

For the given parameters of genetic algorithm initial population is generated. Then, for each chromosome objective function is calculated in accordance with (2). If the initial search ranges are used, the best chromosomes are sought, such whose aim function is less than $\alpha$ If the number of best chromosomes in the population is less than half of the population size, the new generation is created and a new iteration of the inverse problem solution is performed. If the number of the best chromosomes in the population is more than half of the population size $\mathrm{N}_{\mathrm{pop}} / 2$, the new ranges are determined for each parameter. For this case the best chromosomes of generation are used in order to calculate the averages (means) and root-mean-square values for each reconstruction parameter. The obtained estimations allow to use a new range for searching of pavement parameters $\vec{P}_{\min }$ and $\vec{P}_{\max }$. This allowed us to narrow the search range adaptively, and for each electro-physical parameter differently.

In the second stage value $\mathrm{K}$ was set by large, therefore the threshold $\alpha$ was reduced, and then searched the parameters of the $\vec{P}_{M}$ vector. The new generation is formed with the using of new $\vec{P}_{\min }$ and $\vec{P}_{\max }$, and then the next iteration of the inverse problem is performed. However, one of the best individual of generation is sought in the second stage If the value of the aim function $\Phi$ for such chromosome is not more than the value of a threshold $\alpha$, then solving the inverse structure problem is finished. The values of best chromosome parameters are used to determine the vector $\vec{P}_{M}^{*}$, which is the solution of the inverse problem.

\section{Model-Based Investigation of Modified Genetic Algorithm of the Pavement Subsurface Radar Probing Inverse Problem}

\subsection{Basic model of the pavement structure}

The five-layered model of the pavement structure has been used in our investigations. Electrophysical parameters of the model partial layers as well as the parameters of two semi-infinite spaces (upper space - air, and low space - sub-grade) are presented in Table 1. The electro-physical parameters of the pavement structure has been modelled taking into account that the roadway coverage layers are composed of such materials as asphalt, concrete, crushed stone, crushed slag, sand and others.. 
Table 1. Electro-physical parameters of five-layered pavement model

\begin{tabular}{|c|c|c|c|c|}
\hline \multirow{2}{*}{ № } & \multirow{2}{*}{ Partial medium of model } & \multicolumn{3}{|c|}{ Electro-physical parameters of partial model } \\
medium
\end{tabular}

Iterative procedure was used for finding of the aim function $\Phi$ global minimum with help of the modified genetic algorithm in according to block diagram which is shown on Figure 4.

All results were obtained for frequency range 10-500 MHz. To obtain statistical assessment of the reconstruction errors about 100 solutions of the inverse problem were used.

\subsection{Influence of initial search ranges and coefficient $K$ on the errors of the model parameter's reconstruction}

Influence of initial search ranges and coefficient $K$ on the errors of the model parameter's reconstruction is illustrated by Figure 5 and Figure 6, where dependences of average relative displacement and normalized root-mean-square (RMS) values of model parameter's reconstruction are presented.
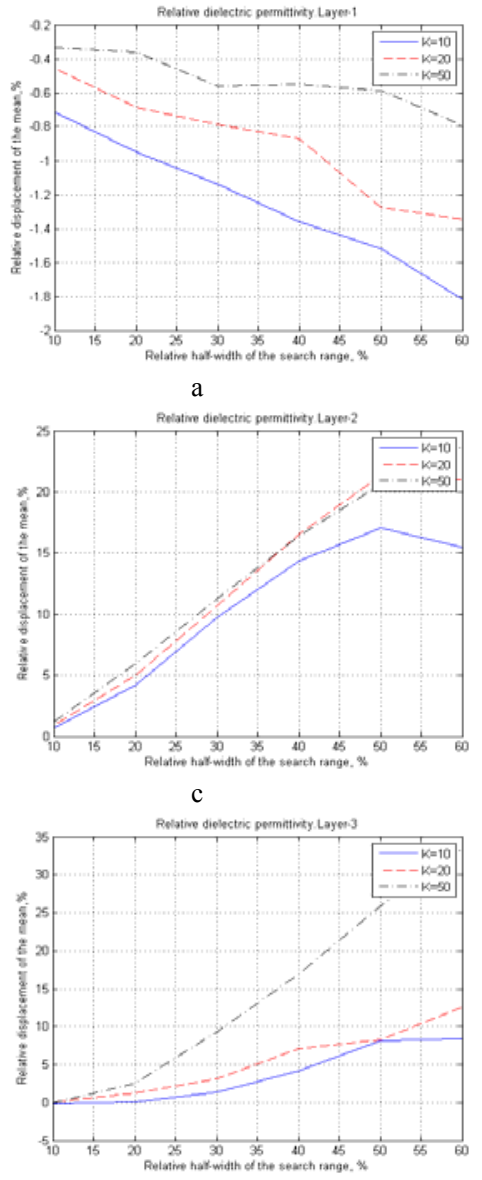

e

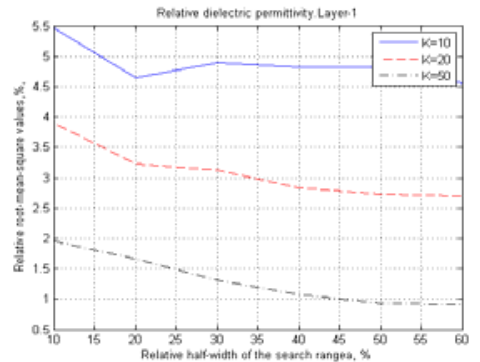

$\mathrm{b}$
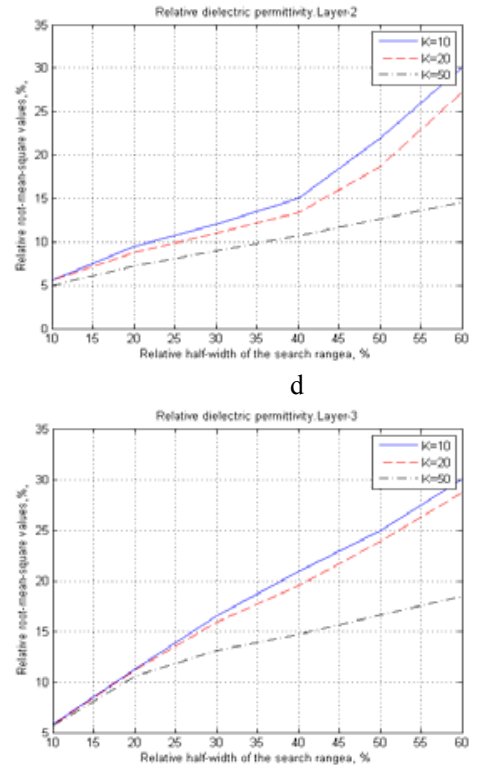

$f$

Figure 5. Influence of width of search range and of value $\mathrm{K}$ on relative displacement (a, c, e) and relative RMS (b, d, f) of constructed values of relative dielectric permittivity of the top tree layers 

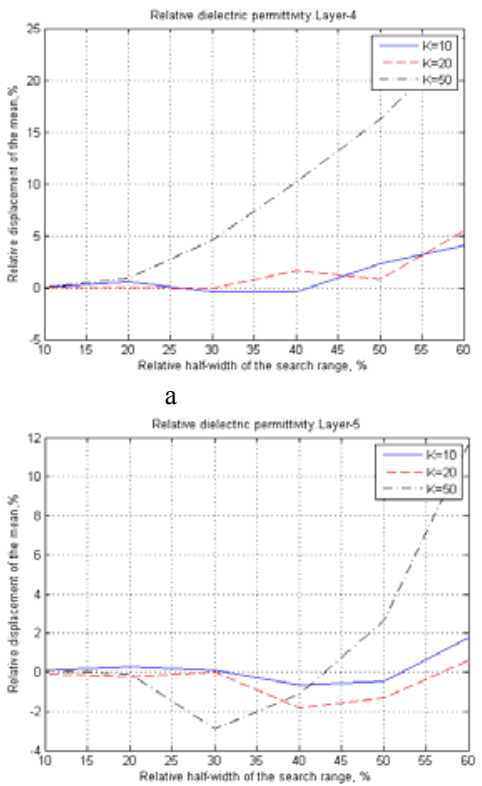

c

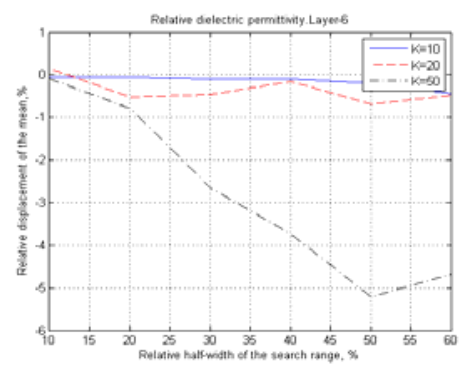

e

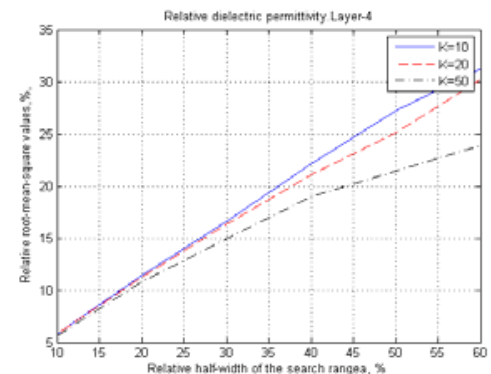

b

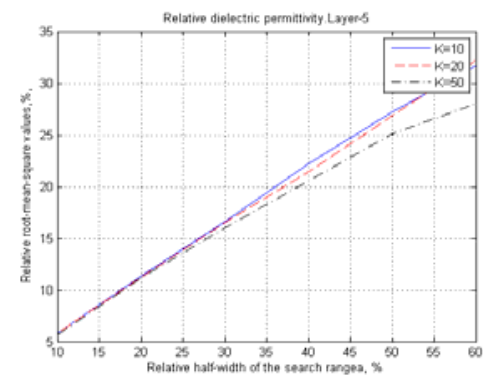

d

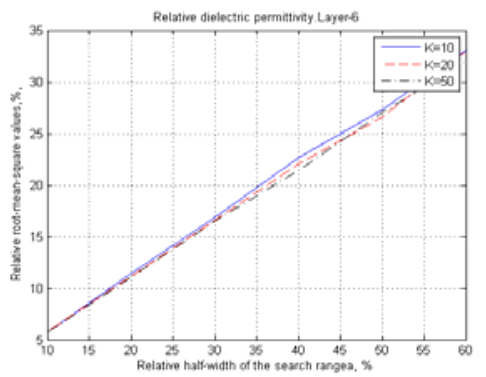

Figure 6. Influence of width of search range and of value $\mathrm{K}$ on relative displacement (a, c, e) and relative RMS (b, d, f) of constructed values of relative dielectric permittivity of the lower tree layers

The average relative displacement of relative dielectric permittivity reconstruction results of the first layer value is less than $2 \%$, the relative RMS slightly depends on K. Displacement and RMS values of reconstructed relative dielectric permittivity of the second and third layers is almost linearly dependent on the width of the search ranges and almost independent of $\mathrm{K}$. Displacement values of reconstructed relative dielectric permittivity of the other layers is independent on the width of the search ranges and close to zero for small values of $\mathrm{K}$, but depends linearly for large values of K. However, the RMS values of these parameters are linearly dependent on the width of the search ranges.

Figure 7 and Figure 8 shows a similar dependence obtained at reconstruction of the layer thickness. The average relative displacement and RMS values of thickness reconstruction results of the first layer linearly increase with increasing of search range and decrease with increasing the coefficient K. Displacement values of reconstructed thickness of the second layer is independent on the width of the search range and close to zero for small values of $\mathrm{K}$. However, the relative displacement increases when $\mathrm{K}$ increases to 50 .

The RMS values of thickness reconstruction results of the second layer are linearly dependent on the width of the search range. Displacement values of reconstructed thickness of the other layers are independent on the width of the search ranges and values of K. Displacements do not exceed 5\%, even if the search rangers are very wide. The RMS values of thickness reconstruction results of the other layers do not depend on $\mathrm{K}$ and depend on the width of the search ranges a perfectly linear.

On Figure 9 we have shown the influence of initial width of search ranges and value $\mathrm{K}$ on average relative displacement (Fig. 9 a, c, e) and normalized RMS values (Fig. 9 b, d, f) of reconstruction results of electrical conductivity of lower layers of roadway coverage. Displacement values of reconstructed electrical conductivity of all layers are independent on the width of the search range and close to zero for all values of $\mathrm{K}$. The RMS values of thickness reconstruction results of the all layers do not depend on $\mathrm{K}$ and depend on the width of the search ranges a perfectly linear. 

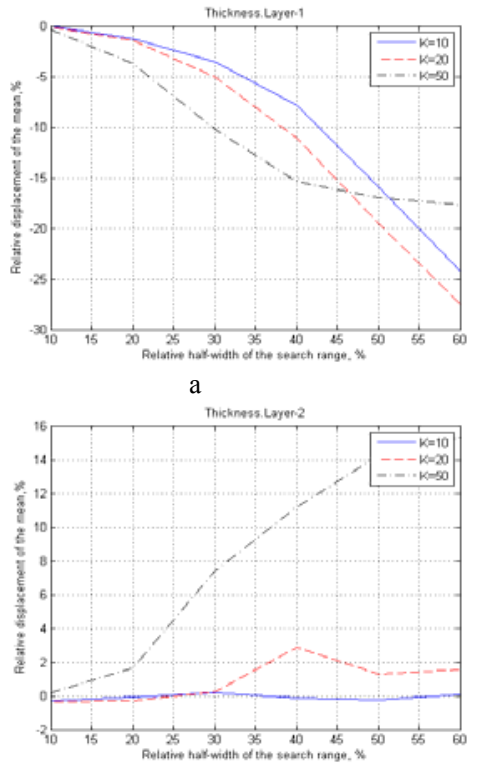

C

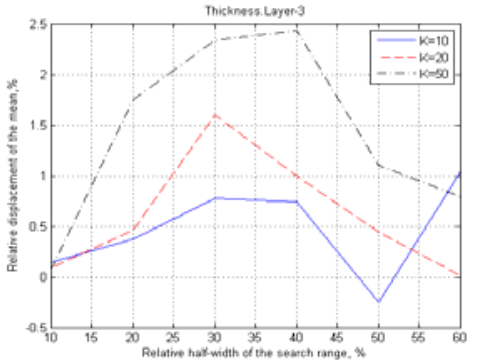

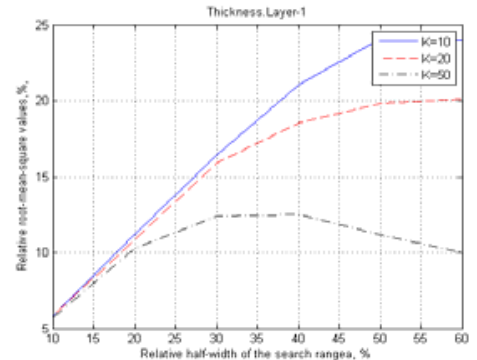

b

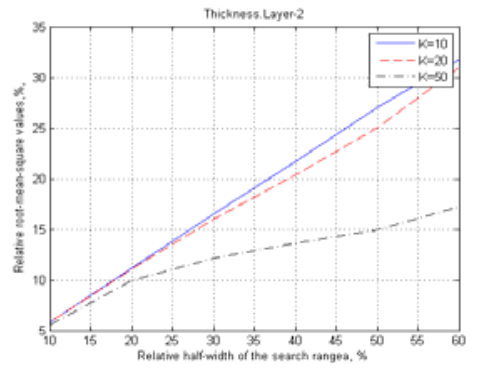

d

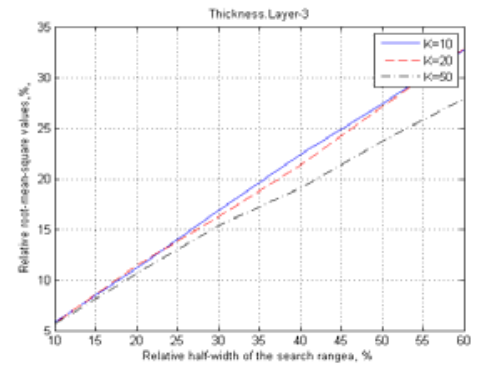

Figure 7. Influence of width of search range and of value $\mathrm{K}$ on relative displacement (a, c, e) and relative RMS (b, d, f) of constructed values of thickness of the top tree layers

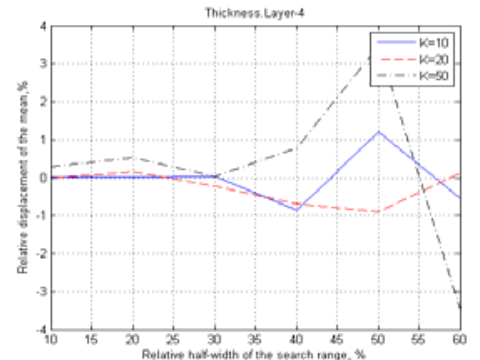

a

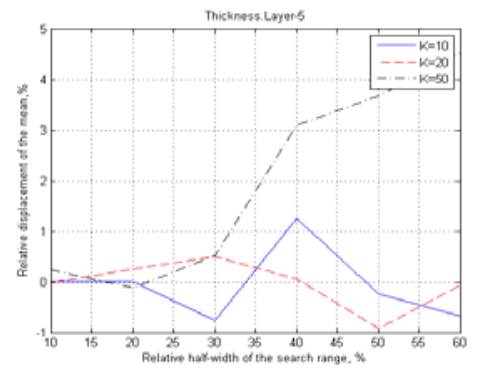

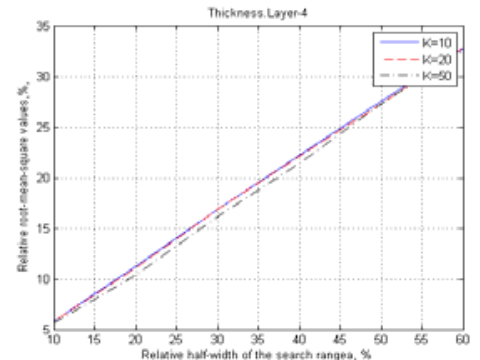

$\mathrm{b}$

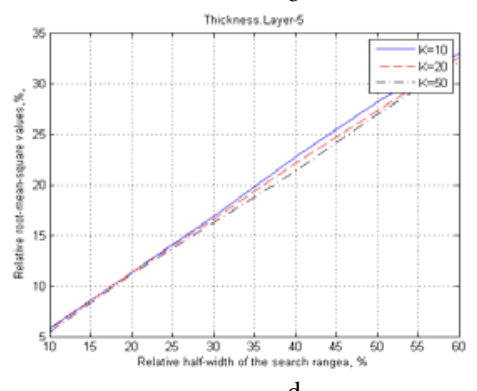

d

Figure 8. Influence of width of search range and of value K on relative displacement (a, c) and relative RMS (b, d) of constructed values of thickness of the lower two layers

Character of the influence of the search range widths and the coefficient $\mathrm{K}$ is explained by to the degree of influence of each electro-physical parameter on the aim function. Figures 10, 11 and 12 show the cross section of the aim function along all electro-physical parameters (directions). 

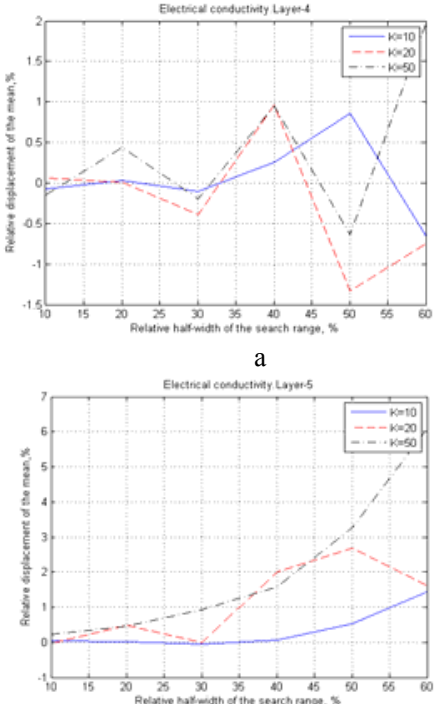

$\mathrm{c}$

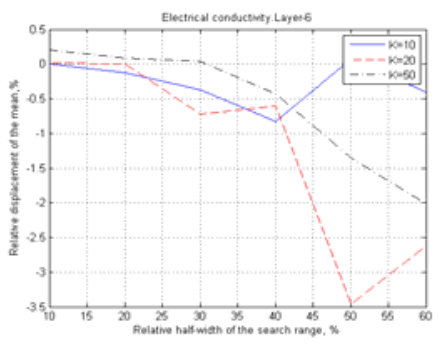

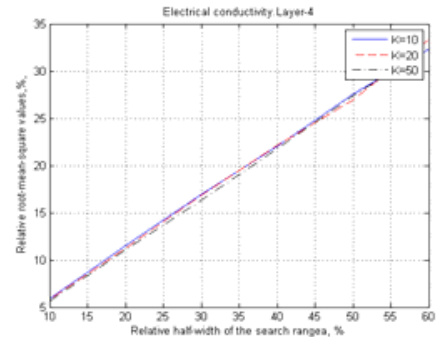

b
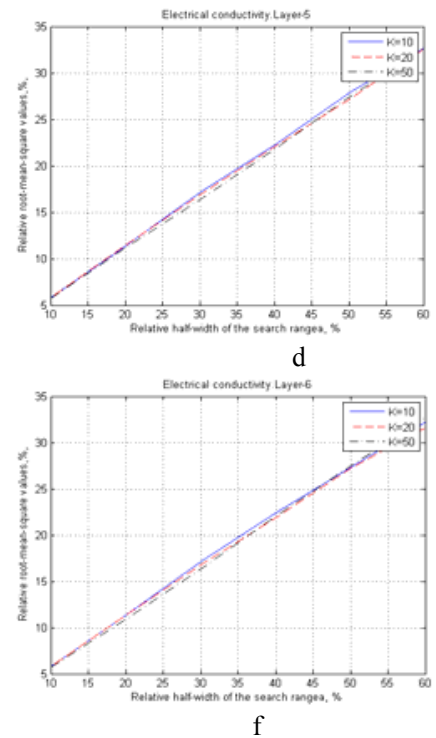

Figure 9. Influence of width of search range and of value $\mathrm{K}$ on relative displacement (a, c, e) and relative RMS (b, d, f) of constructed values of electrical conductivity of the lower tree layers

The cross-sections of the aim function along the relative dielectric permittivity of the all layers are asymmetrical (Figure 10). The aim function increases more slowly, if the value of the relative dielectric permittivity of each layer increases with respect to the model value. Degree of asymmetry of the cross sections is different. The cross - section along the relative dielectric permittivity of the second and third layers have the greatest asymmetry. The asymmetry of the aim function with respect to the model values of the relative dielectric permittivity is causing displacement of the reconstructed relative dielectric permittivity of the first three layers (Fig. 9 a, c, e).

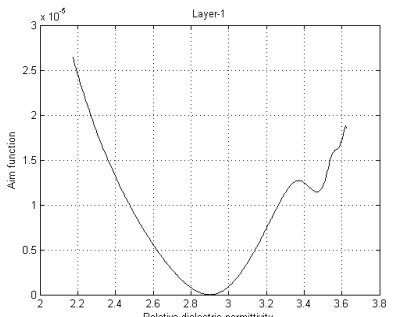

a

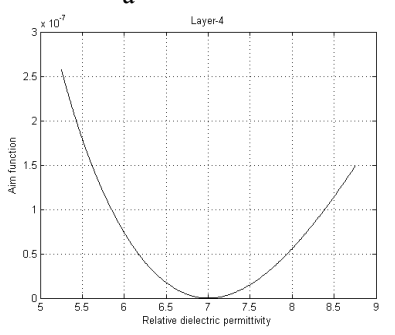

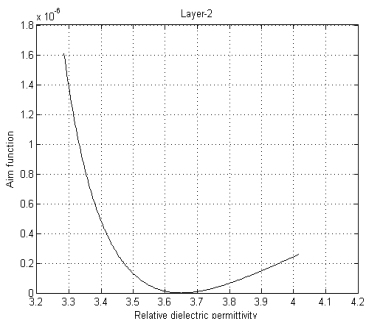

$\mathrm{b}$

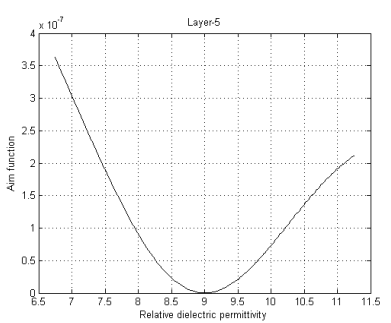

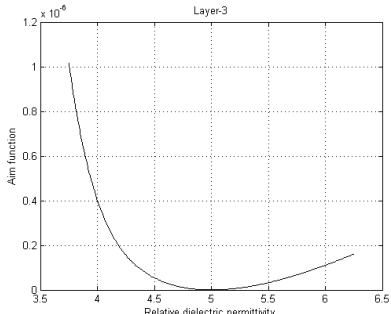

c

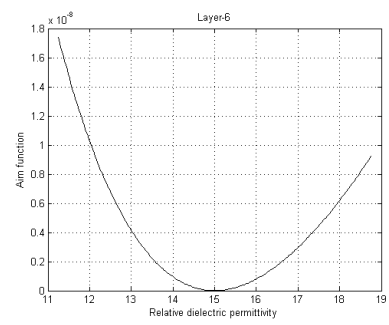

f

Figure 10. Model-based cross - sections of the aim function along the relative dielectric permittivity of pavement layers: $\mathrm{a}$-1-st layer; $\mathrm{b}-2$-nd layer; $\mathrm{c}-3$-rd layer; $\mathrm{d}$ - 4-th layer; $\mathrm{e}-5$-th layer; $\mathrm{f}-6$-th layer 
The cross-sections of the aim function along the thickness of the all layers are symmetrical (Fig. 11), except for the cross-section along the thickness of the first two layers (Fig. $11 \mathrm{a}, \mathrm{b}$ ). The asymmetry of the aim function with respect to the model values of the thickness is causing displacement of the reconstructed thickness of the first and second layers (Fig. 6 a, c). The local maximums and local minimums are presented at the cross sections of the aim function along the thickness of the bottom two layers (Fig. $11 \mathrm{~d}$, e). This can be explained by large thicknesses of these layers.

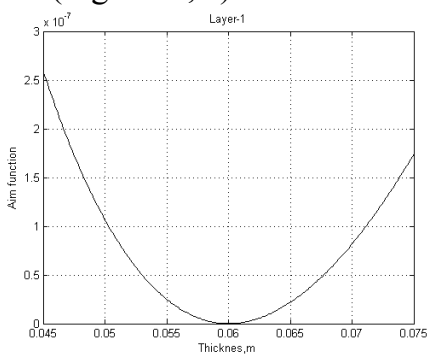

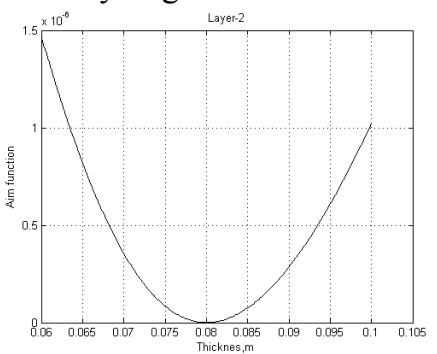

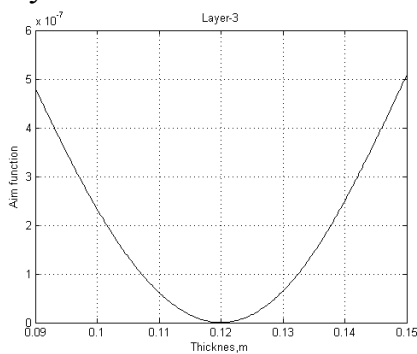

c

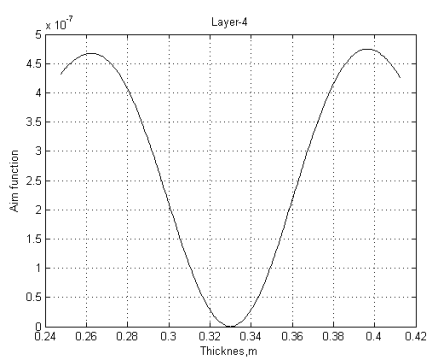

d

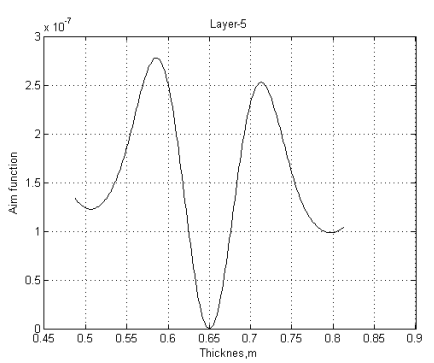

e

Figure 11. Model-based cross - sections of the aim function along the thickness of pavement layers: a - 1-st layer; b - 2-nd layer; $\mathrm{c}-3$-rd layer; $\mathrm{d}$ - 4-th layer; e -5-th layer

The cross-sections of the aim function along the electrical conductivity of the all layers are perfectly symmetrical for all layers as it is shown on Figure 12.

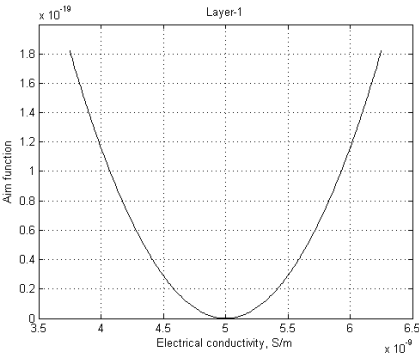

a

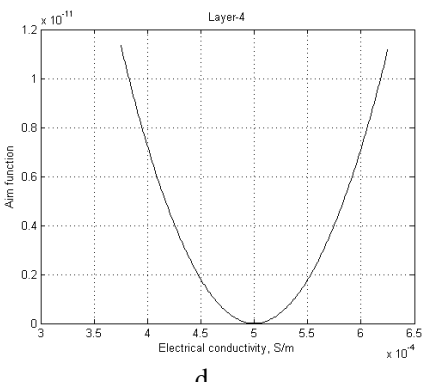

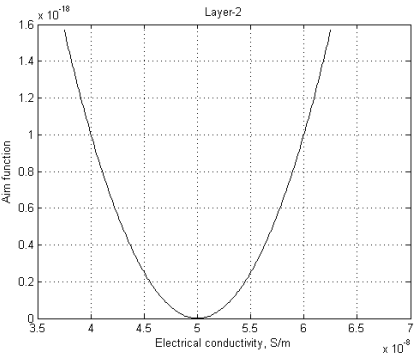

$\mathrm{b}$

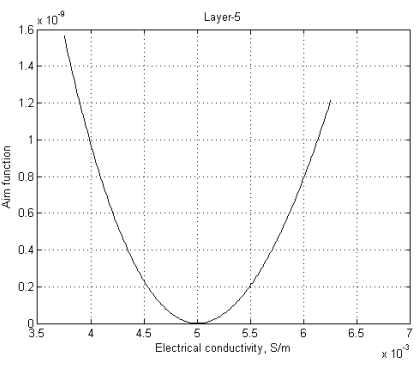

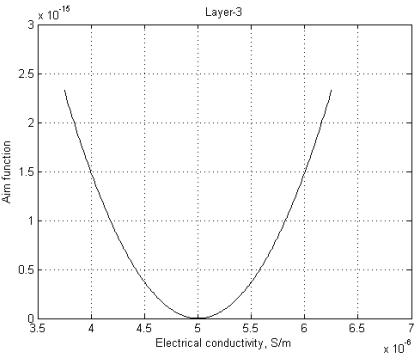

$\mathrm{c}$

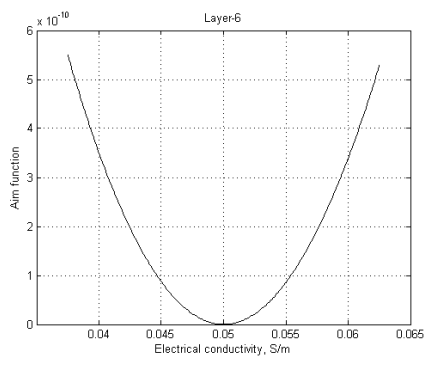

f

Figure 12. Model-based cross - sections of the aim function along the electrical conductivity of pavement layers: $\mathrm{a}$ - 1-st layer; $\mathrm{b}$ - 2-nd layer; $\mathrm{c}$ - 3-rd layer; $\mathrm{d}$-4-th layer; e - 5-th layer; $\mathrm{f}-6$-th layer

The maximum values of the aim function for each section characterize the sensitivity to changes in the aim function of each parameter (for each parameter). The aim function is most sensitive to changes in the relative dielectric permittivity of the first layer. This explains the independence of the relative 
displacement and the normalized RMS of the reconstructed values of the search range width and the coefficient K (Fig. 5 a, b). The aim function is low sensitive to the changing in electrical conductivity. The low sensitivity of the aim function to the changing any of parameters is shown in a linear dependence of the normalized RMS of the search range width (Fig. 6 b, d, f; Fig. 7 b, d, f; Fig. 8 b, d, f; Fig. 9 b, d, f). The linearity of these dependences shows that the set of reconstructed parameter values are distributed in a uniform law. Therefore, the relative average displacement of these parameters is close to zero. The width of the new search range is determined by using of RMS dependences.

If the cross section of the aim function along the electro-physical parameter is asymmetric, the growth of $\mathrm{K}$ increases the relative average displacement of the reconstruction value of this parameter (Fig. 4-Fig. 7).

In order to define the optimal value $\mathrm{K}$ we researched the influence of relative width of the initial search range and coefficient $\mathrm{K}$ on generation amount (iteration of GA) to change the width of the search ranges and on number of the best individuals (chromosomes) are used to determine the displacement and RMS of reconstructed values of electro-physical parameters. The obtained dependences are shown on Figure 12. The population size was about 100; therefore, the definition of new search ranges was performed after the generation of at least the 50 best individuals in the population.

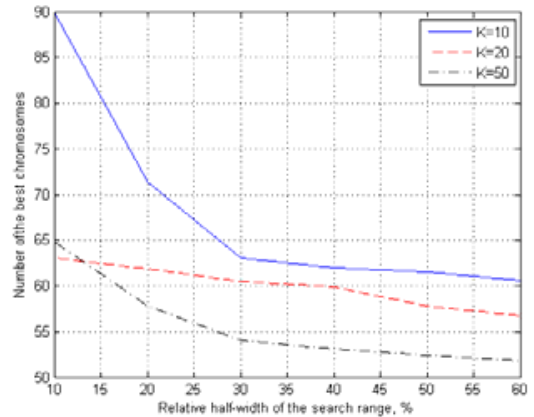

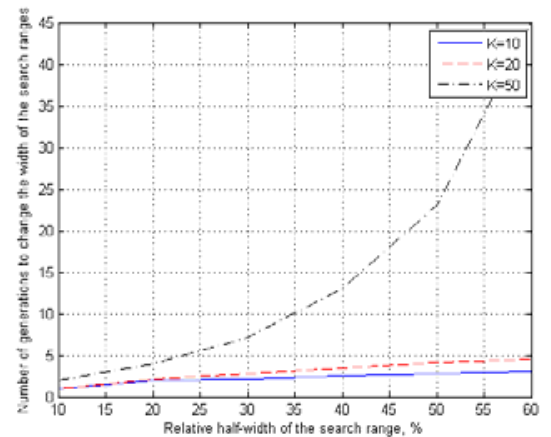

b

Figure 12. Influence of the conditions for solving of the model-based inverse problem on: a - generation amount; $\mathrm{b}-$ the best individuals in genetic population

We see that for $\mathrm{K}=50$ the generation amount of increases significantly, but an increase in $\mathrm{K}$ does not reduce the relative displacement and RMS of reconstructed values (Fig. 4 - Fig. 8). Value K was selected in the range of $15 \ldots 30$.

\subsection{Improving the iterative procedure to solve radar inverse problem with the adaptive narrowing parameter's search range}

To improve the algorithm for solving of the inverse problem with adaptive narrowing of the search range necessary:

- to perform analysis of the distribution of values in the search ranges after the formation of the specified number of the best individuals;

- to define new search range for those parameters only that have irregular distribution of values in the search range;

- to define new search range taking into account displacement and RMS of reconstructed parameter;

- to define threshold $\alpha$ as the mean value of the aim functions of the best individuals after each refinement of search ranges;

- after refinement search range to determine the best individuals, and then the electro-physical parameter's values, which have been found the best individuals, to use for calculate the average and RMS values of the electro-physical parameter, and calculated values are considered reconstruction values;

- to set the number of the best individuals of at least 100 to ensure that the conditions of the statistical processing of the results.

To do this, the following changes and additions were made to the algorithm

1. The value of $\mathrm{K}$ was chosen to be 20 , and possible displacements of the reconstructed relative dielectric permittivity of all the layers are defined except the first layer and possible displacements of the reconstructed thickness the first three layers.

2. If the condition is "the number of the best individuals of more than half the size of the population" (at least 100) is satisfied, the average, RMS, coefficient of excess, coefficient of variation and 
the normalized RMS are determined using electro-physical parameter's values, found the best individuals. RMS normalized relative half-width of the search range.

3. If the normalized RMS of electro-physical parameter is less than 0.2 (the values, found the best chromosomes, not distributed in a uniform law), we calculated a new search range for this parameter.

4. The middle of the new interval is calculated using the average, standard deviation and coefficient of excess. The displacement is used only for the first refining the search range. Further width and boundaries of the new search range are calculated with using RMS and coefficient of excess.

5. After finding a new search range for at least one electro-physical parameters, the new threshold value $\alpha$ is calculated equal to the average value of the aim function of the best individuals. The new value $\alpha$ used in the next iteration to determine the best individuals.

6. If the search range for a parameter is performed with a coefficient of variation of less than 0.03 , the definition of the new search ranges for this parameter is terminated. This condition reduces the probability that a new search range does not contain the true value of electro-physical parameter.

7. The calculation of the new search ranges is complete when calculating new search ranges for the relative dielectric permittivity and thickness of the all layers, or performing a specified number of iterations of the algorithm.

After determining the new search range a new generation of individuals is formed, the best individuals are identified and the electro physical parameter's values found best individuals are averaged. The averaged values of the electro-physical parameter are the solution of the inverse problem.

In order to evaluate the algorithm, we determined the narrowing coefficients that characterize the narrow of search ranges. Figure 14 shows the values of these coefficients for the relative dielectric permittivity and thickness of the layers.
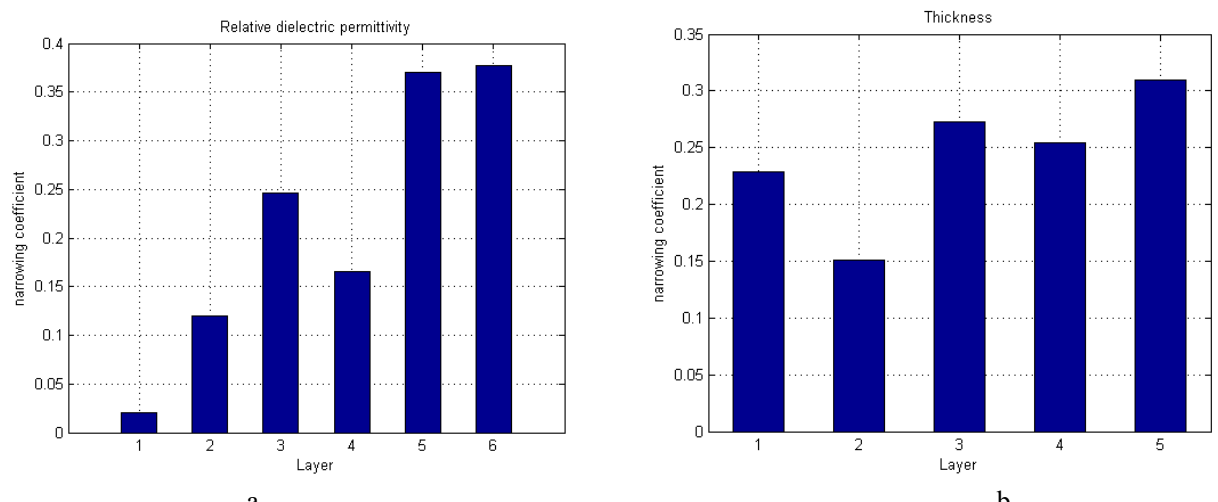

Figure 14. Coefficients of the narrowing of search ranges for model-based pavement electro-physical parameter's reconstruction by modified genetic algorithm with the adaptive narrowing parameter's search range: a coefficients of the narrowing for layer's relative dielectric permittivity; $b$ - coefficients of the narrowing for layer's thickness

An important feature of the algorithm is the probability of finding the model values of electrical parameters in the new narrowed search ranges (probability of localization). Figure 15 shows the probabilities of localization for model-based pavement electro-physical parameters in the new narrowed search range.

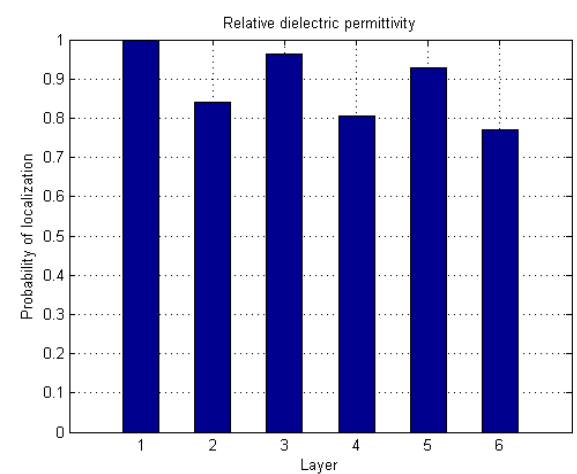

a

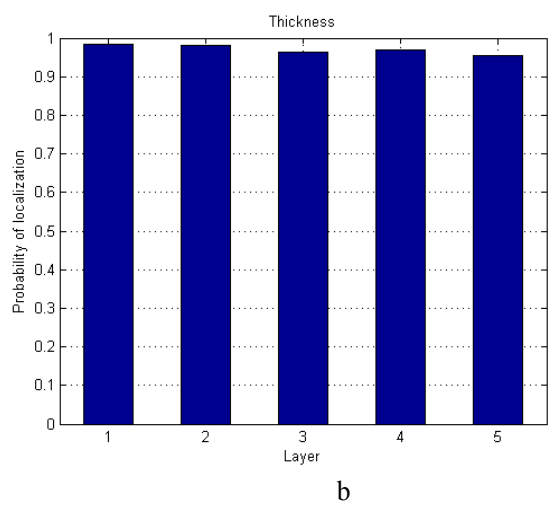

Figure 14. Probability of localization for model-based pavement electro-physical parameters in the new narrowed search range: a - probability of layer's relative dielectric permittivity localization; b - probability of layer's thickness localization 
High localization probability of model values provides decrease errors of model parameters reconstruction. Figure 16 shows the relative errors of model parameters reconstruction after narrowing the initial search ranges.

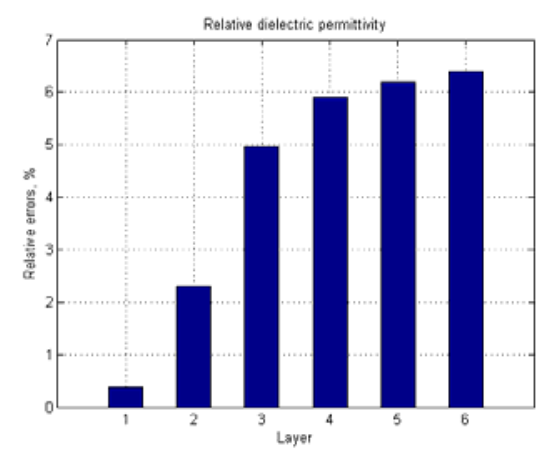

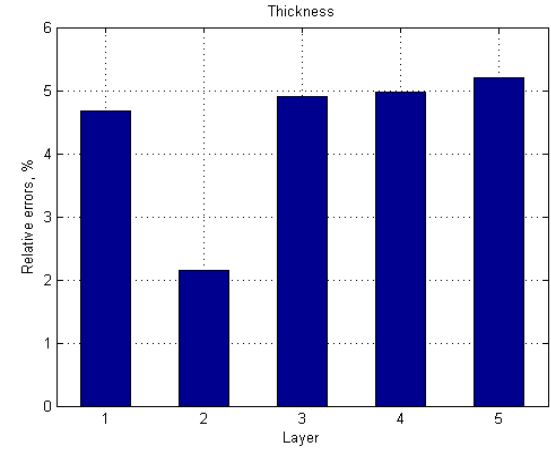

Figure 15. Relative errors of model-based pavement electro-physical parameter's reconstruction by modified genetic algorithm with the adaptive narrowing parameter's search range: a-results reconstruction of layer's relative dielectric permittivity; $\mathrm{b}$ - results reconstruction of layer's thickness

The results were obtained when the relative half-width of the initial search ranges were equal to $25 \%$. At such initial search ranges to narrow of range number of iterations are not greater than 10 . The search range of first layer relative dielectric permittivity is specified at the first iteration always. This increases the sensitivity of the aim function to change of other parameters and allows to narrowing the search ranges for relative dielectric permittivity and thickness of other layers. However, narrowing the search range for electrical conductivity was not made.

\section{Conclusions}

To obtain near-optimal solutions of GPR inverse problem in reasonable running times it is proposed to use a modified genetic algorithm with the adaptive narrowing parameter's search range. Model-based investigation of this algorithm for five-layered pavement is performed to receive following results:

- using the characteristics of the distribution of values in the search ranges can be consistently narrow the search ranges of electro-physical parameters;

- adaptive narrowing of search ranges increases the sensitivity of the aim function to changes of other parameters of the layers;

- using adaptive algorithm of search ranges parameters is possible to narrow the search ranges reconstruction of parameters and the duration of the inverse problem solving.

\section{References}

1. Pavement Types Module-Pavement Interactive. http://pavementinteractive.org/php?title=HMA_ Pavement.

2. Georgopoulos, A., Loizos, A. \& Flouda, A. (1995). Digital image processing as a tool for pavement distress evaluation. Journal of Photogrammetry and Remote Sensing, 50(1), 23-33.

3. Grote, K., Hubbard, S., Harvey, J., \& Rubin, Y. (2005). Evaluation of infiltration in layered pavements using surface GPR reflection techniques. Journal of Applied Geophysics, Journal of Applied Geophysics, 57, 129-153.

4. Krainyukov, A., Kutev, V., Opolchenov, D. (2010). Reconstruction of the Roadway Inner Structure Electro-physical Characteristics. In Proceedings of the 10th International Conference "Reliability and Statistics in Transportation and Communication (RelStat'10), October 20-23, 2010 (p. 55; pp. 382-393). Riga: Transport and Telecommunication Institute.

5. Krainyukov, A., Kutev, V. (2011). Using of radar monitoring for road coverage quality estimation. In Proceedings of the 11th International Conference "Reliability and Statistics in Transportation and Communication (RelStat'11), October 19-22, 2011 (pp. 275-283). Riga: Transport and Telecommunication Institute.

6. Journal "Perspective Information Technologies and Intelligent Systems" (In Russian) - http://pitis.tsure.ru/.

7. Krainyukov, A., Kutev, V. (2011). Problems of road coverage quality estimation by GPR probing method. Transport and Telecommunication, 12(4), 4-13. 